\title{
GAS PRODUCTION RATES IN COMETS
}

\author{
A.A. DE ALMEIDA \\ Department of Astronomy, Inst. of Astronomy and Geophysics, \\ University of São Paulo \\ C.P. 9638, CEP 01065, São Paulo - Brazil
}

\begin{abstract}
Emission fluxes of $C N, C_{2}$ and $C_{3}$ carbon-bearing molecular species observed in the coma of comets Bennett $(1969 i \equiv 1970 I I)$, West $(1975 n \equiv 1976 \mathrm{VI})$, $P /$ Halley (1982i), Hartley-Good (1985l) and Bradfield (1987s) are analysed in the framework of Haser model. $C N, C_{2}$ and $C_{3}$ production rates are determined using recently derived fluorescence efficiencies. The dependence of $C N, C_{2}$ and $C_{3}$ production rates on the heliocentric distance and the possible correlations among these radicals is studied and briefly discussed.
\end{abstract}

\section{INTRODUCTION}

As a part of the systematic programme - Determination of Gas and Dust Production Rates in Comets (GDPC) - going on in this laboratory (Almeida et al., 1989; Almeida, 1991), the emission band fluxes of cometary $\mathrm{CN}, \mathrm{C}_{2}$ and $\mathrm{C}_{3}$ carbon bearing molecular species observed in the coma of comets Bennett (1969i $\equiv 1970 \mathrm{II})$ (Babu and Saxena, 1972) West (1975n $\equiv 1976$ VI) (Sivaraman et al., 1979), P/Halley (1982i) (Goraya et al., 1989a; 1989b), Hartley-Good (1985 $\ell$ ) (Rautela et al., 1989) and Bradfield (1987s) (Rautela and Sanwal, 1988; Ojha and Joshi, $1989)$ are analysed. These comets are studied in the framework of the Haser model (1957) by using recently derived fluorescence efficiencies (g-factors) at $r_{h}=1 \mathrm{AU}$ (Almeida et al., 1989) and appropriate numerical parameters which are known for the more prominent species $\mathrm{CN}, \mathrm{C}_{2}$ and $\mathrm{C}_{3}$ (Cochran, 1985). Haser model analysis of $\mathrm{CN}, \mathrm{C}_{2}$ and $\mathrm{C}_{3}$ production rates are developed for these comets and the possible logarithimic correlations among these molecular species and comets graphically analysed.

\section{DISCUSSION}

The derived $\mathrm{CN}, \mathrm{C}_{2}$ and $\mathrm{C}_{3}$ production rates using recently derived fluorescence efficiencies (Almeida et al., 1989) may have a systematic error amounting to about \pm 20 percent and where possible these results were compared with the ones found in the literature. Equations 1 of Almeida et al. (1989) and Konno and Wyckoff (1989), derived independently, are equivalent within this uncertainty.

In the particular case of West $(1975 \mathrm{n} \equiv 1976 \mathrm{VI})$ since the comet broke up in four different parts $(\mathrm{A} \longrightarrow \mathrm{D}: 1976$ Feb. $19.1 \pm 0.2 ; \mathrm{A} \longrightarrow \mathrm{B}: 1976$ Feb. $27.7 \pm 0.2$; 
$\mathrm{A} \longrightarrow \mathrm{C}: 1976$ Mar. $6.5 \pm 0.3$ (where $\mathrm{A}$ is the principal fragment and B, C, D are secondary fragments (Sekanina, 1982)), the expected errors should be at least twice as much since the data used (Sivaraman et al., 1979) corresponds to observations performed after the primary nucleus (A) of the comet had splited in these four fragments close to perihelion passage (February 25.22, 1976 (UT)). Surprisingly the results for comet West $(1975 \mathrm{n} \equiv 1976 \mathrm{VI})$ correlates very well with the ones obtained for the other four comets (see for instance Figure 2), and particularly with comet $\mathrm{P} /$ Halley (1982i) for $\mathrm{CN}$ and $\mathrm{C}_{2}$. This might suggest that Sivaraman et al. (1979) tracked mainly the principal fragment $(A)$ of the nucleus during their observations.

From Figures 1 to 4 one can easily conclude that as far as the global production rates are concerned it decreases for $C N$ and $C_{2}$ and increases for $C_{3}$ with the heliocentric distance. Hartley-Good $(1985 \ell)$ is the comet that shows the least $\mathrm{C}_{3}$ production rate, compared to $\mathrm{CN}$ and $\mathrm{C}_{2}$ among the five comets considered in this study.

Acknowledgments: The author is thankful to Mr. Julio C. Klafke for efficient assistance in plotting the graphs and to $\mathrm{CNPq}$ for financial support under grant No. 306304/88-0.
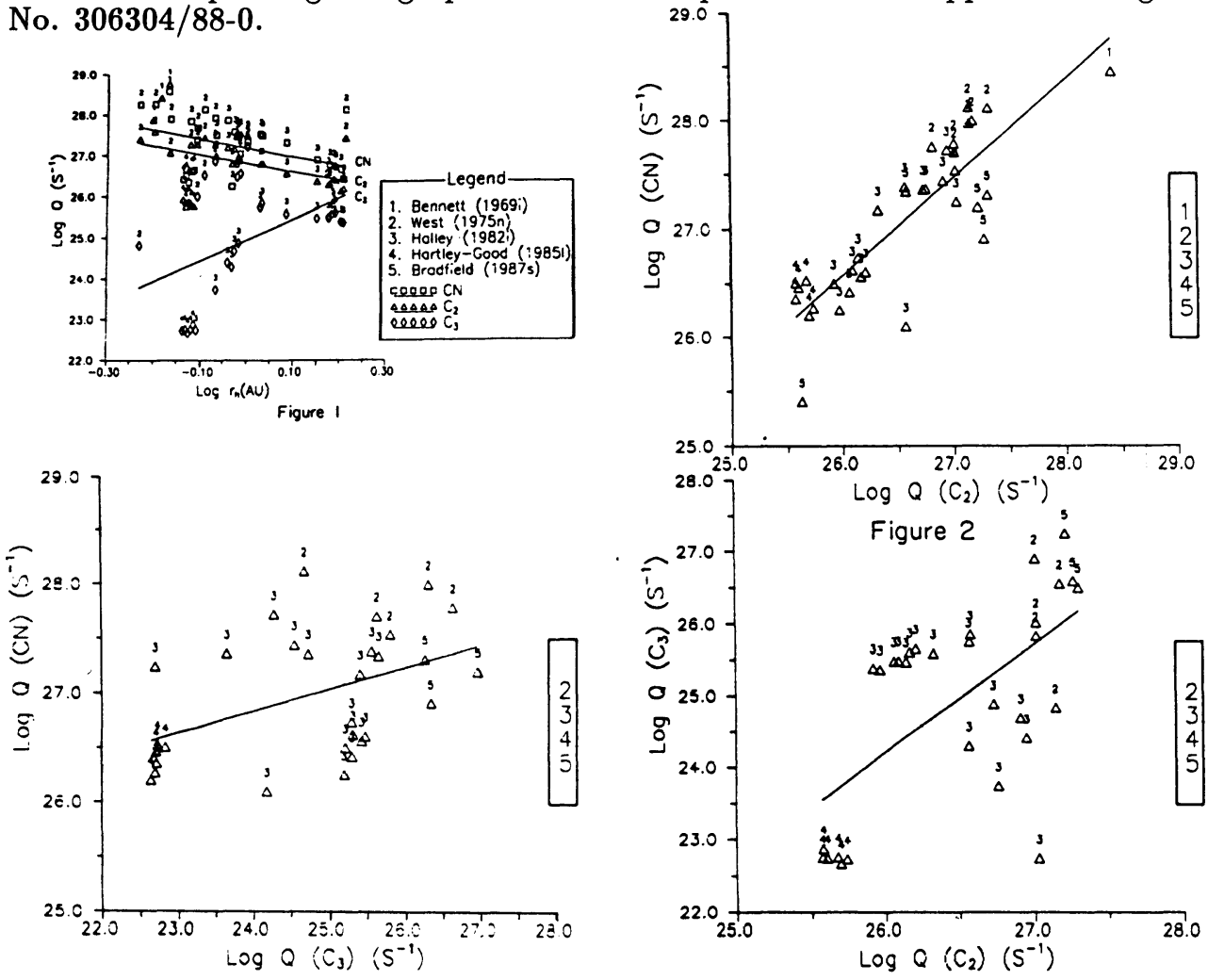

Figure 3

Figure 4 
Figure 1 - Global production rates of $\mathrm{CN}, \mathrm{C}_{2}$ and $C_{3}$ molecules as a function of heliocentric distance for comets, Bennett (1969i $\equiv 1970 \mathrm{II})$, West $(1975 n \equiv 1976 \mathrm{VI})$, $\mathrm{P} /$ Halley (1982i), Hartley-Good (1985 $\ell$ ) and Bradfield (1987s). The straight lines represent the logarithimic correlations.

Figure 2 - Logarithimic correlation between production rates of $\mathrm{CN}$ and $\mathrm{C}_{2}$ molecules for comets Bennett (1969i $\equiv 1970 \mathrm{II})$, West $(1975 n \equiv 1976 \mathrm{VI}), \mathrm{P} / \mathrm{Halley}$ (1982i), Hartley-Good (1985 $\ell$ ) and Bradfield (1987s).

Figure 3 - Logarithimic correlation between production rates of $\mathrm{CN}$ and $\mathrm{C}_{3}$ molecules for comets Bennett (1969i $\equiv 1970 \mathrm{II})$, West $(1975 n \equiv 1976 \mathrm{VI}), \mathrm{P} / \mathrm{Halley}$ (1982i), Hartley-Good (1958 $\ell$ ) and Bradfield (1987s).

Figure 4 - Logarithimic correlation between production rates of $\mathrm{C}_{2}$ and $\mathrm{C}_{3}$ molecules for comets Bennett (1969i $\equiv 1970 \mathrm{II})$, West $(1975 n \equiv 1976 \mathrm{VI}), \mathrm{P} / \mathrm{Halley}$

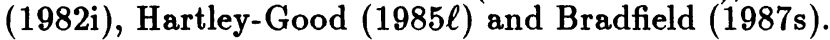

\section{References}

Almeida, A.A., Singh, P.D. and Burgoyne, C.M. (1989) 'Haser Model CN, $\mathrm{C}_{2}$ and $\mathrm{C}_{3}$ Production Rates in Some Comets', Earth, Moon and and Planets 47, 15-31.

Almeida, A.A. (1991), 'An Analysis of the Spectrophotometric and Photometric Observations of Comets 1967II, 1968I, 1968V, and 1968VI', Earth, Moon and Planets (in press).

Babu, G.S.D. and Saxena, P.P. (1972), 'Spectrophotometry of Comet Bennett', Bull. Astron. Inst. Czech 23, 346-349.

Cochran, A.L. (1985), 'A Re-Evaluation of the Haser Model Scale Lengths for Comets', Astron. J. 90, 2609-2614.

Goraya, P.S., Sanwal, B.B., Rautela, B.S., Duggal, H.K. and Malhi, J.S. (1989a), 'Spectrophotometry of P/Halley (1982i)', Earth, Moon and Planets 44, 243-249.

Goraya, P.S., Rautela, B.S., Sanwal, B.B., Gupta, S.K., Duggal, H.K., and Malhi, J.S. (1989b), Spectrophotometric Study of Periodic Comet Halley (1982i)'Earth, Moon and Planets 45, 17-27.

Konno, I. and Wyckoff, S. (1989), 'Atomic and Molecular Abundances in Comet Giacobini-Zinner', Adv. Space Res. 9, 163-168.

Ojha, D.K. and Joshi, S.C. (1989), 'Spectrophotometry of Comet Bradfield (1987s)', Earth, Moon and Planets 44, 1-5.

Rautela, B.S. and Sanwal, B.B. (1988), 'Spectrophotometric Study of Comet Bradfield (1987s)', Earth, Moon and Planets 43, 221-225.

Rautela, B.S., Goraya, P.S., Sanwal, B.B., and Gupta, S.K. (1989), 'Spectropho-

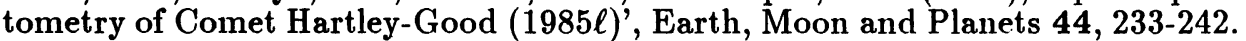

Sekanina, Z. (1982), 'The Problem of Split Comets in Review'in L.L. Wilkening (ed.), Comets, The University of Arizona Press, Tucson, pp. 251-287.

Sivaraman, K.R., Babu, G.S.D., Bappu, M.K.V. and Parthasarathy, M. (1979), 'Emission band and continuum photometry of Comet West (1975n)-I. Heliocentric dependence of the flux in the emission bands and the continuum', Mon. Not. R. ast. Soc. 189, 897-906. 